\title{
Comparative Study on Seed Production Potentiality of Selected Rice (Oryza sativa L.) Hybrid Combinations
}

\author{
M H Rahman'1, M J Hasan¹, M A Bari² and M A Haque ${ }^{3^{*}}$
}

\begin{abstract}
The seed production potentialities of selected 20 hybrid rice combination were assessed in this study. Higher seed yield potentiality and earliness in maturity were two of the most important indicators for popularizing hybrid rice variety in Bangladesh. The field performance of the selected hybrid rice combinations were found to vary significantly for different traits except number of total spikelets per panicle showed insignificant variation among the hybrid rice genotypes. The highest number of tillers per plant was recorded in BRRI7A/BRRI31R (17.33) which was followed by BRRI10A/BRRI20R (16.33) and BRRI7A/BRRI20R (16.17). Maximum number of panicles per plant was produced by BRRI7A/BRRI31R (14.00) which was followed by BRRI10A/BRRI31R (13.07), and BRRI11A/BRRI31R (12.47). The highest number of filled spikelets per panicle was produced by BRRI7A/BRRI31R (48.20) which was followed by IR79156A/BRRI31R (47.83) and BRRI7A/BRRI10R (44.63) respectively. The highest value for percent out crossing rate (\%OCR) was observed in the combination of BRRI7A/BRRI31R (50.23\%) which was followed by BRRI10A/BRRI10R $(46.40 \%)$. The highest value for grain yield per plant was recorded in BRRI7A/BRRI31R $(8.17 \mathrm{~g}$ ) followed by BRRI10A/BRRI10R (7.85 g) and BRRI11A/BRRI15R (7.68 g), respectively. The lowest value for grain yield per plant was found in IR79156A/BRRI31R (3.97 g). Considering comparative study of floral traits, BRRI7A/BRRI31R hybrid rice combination has good commercial prospects but seed production potentiality under Bangladesh conditions needs to be estimated with fine tune.
\end{abstract}

Key words: Out crossing rate, seed production, potentiality and combinations

\section{INTRODUCTION}

Rice is arguably the most important staple food crop in the world. More than half of the world's population consumes rice as their primary source of food, and will almost certainly continue to use rice as the main diet in the future. The most remarkable achievement of Bangladesh since her independence in 1971 is the acceleration of rice production, enabling her self-sufficiency in food production rather than an import dependent one. The production of food grain was 8.74 million metric tons (MMT) in 1971-72 which increased to 34.70 million metric tons in 2014-15 (BBS, 2018).

Hybrid rice technology is one of the most talked issues in our country where strengthening food security is a priority agenda. Feeding the expanding population from the decreasing cultivable land is a great challenge to our agriculture. Moreover, climate change and bio-energy have added extra challenges to food security issue. How to solve this problem? The only option is to expand vertically to balance the equation, food = people. Hybrid rice, as it has the capability to increase yield by $15-20 \%$ more than even the best HYV, can be an answer for vertical expansion. Vertical increase in yield can meet the food demand of expanding population. There is a gradual development of hybrid rice cultivation in our country since 2001-2002 to 2016-17. The area planted to hybrid rice in the country during Boro 2016-17 was around 0.7 Mha has contributed 3-4 MT of additional rice

${ }^{1}$ Hybrid Rice Division, BRRI; 2 and3* Genetics and Plant Breeding Division, BAU, Mymensingh. ${ }^{*}$ Corresponding auther's E-mail: ashraf_gpb2000@yahoo.com 
to the total rice production of the country (AIS, 2018).

Therefore, to break the present yield ceiling of semi-dwarf modern varieties, hybrid rice seems to be an attractive viable alternative if suitable parental lines and effective seed production infrastructure are developed in the public, private, or NGO sectors of the country as well as seed production units should be closely linked with its hybrid rice research units to expedite the transfer of this technology. Therefore, these experiments have been taken for selection of suitable hybrid combination for development of effective seed production ability. So the objective of the research programme was selection of suitable hybrid combination and modifications of the currently used strategies for $F_{1}$ hybrid seed production.

\section{MATERIALS AND METHODS}

The experiment was conducted at the experimental farm of Bangladesh Rice Research Institute (BRRI), Gazipur during November to May 2017-18. The soil of the experimental field was clay loam in texture having $\mathrm{pH}$ of 6.2. It belongs to the Chitra soil series of red brown terrace. Five cytoplasmicgenetic male sterile (CMS) lines (IR75608A, IR79156A, BRRI7A, BRRI10A and BRRI11A) and four (BRRI10R, BRRI15R, BRRI20R and BRRI31R) restorer lines were used. Twenty seed production combinations (IR75608A/RRI10R, IR75608A/BRRI20R, IR79156A/BRRI10R, IR79156A/BRRI20R, BRRI7A/BRRI10R, BRRI7A/BRRI20R, BRRI10A/BRRI10R, BRRI10A/BRRI20R, BRRI11A/BRRI10R, BRRI11A/BRRI20R and BRRI11A/BRRI31R) were used in this study. The seed of these parental lines were collected from Hybrid rice division of BRRI. Seeds of all genotypes were soaked separately following BRRI prescribed duration gap between $\mathrm{A}$ and $\mathrm{R}$ lines for 48 hours in clothes bag. Soaked seeds were picked up from water and wrapped with straw and gunny bag to increase the temperature for facilitating germination. The germinated seeds were sown in seed bed for raising seedlings. The land was prepared thoroughly by 3-4 times ploughing and cross ploughing followed by laddering to obtain a good puddled condition.

The experiment was laid out in a randomized complete block design (RCBD) with three replications. The unit plot size was $4 \mathrm{~m} \times 2 \mathrm{~m}$. Fertilizer were applied @ 270, 130, 120,70 , and $10 \mathrm{~kg} /$ ha of urea, TSP, MOP, zinc sulphate and gypsum, respectively. Total urea was applied in three installments at 15, 35 and 55 days after transplanting (DAT). One third of MOP was applied with $2^{\text {ndtop }}$ dressing of urea. Thirty-day-old seedlings of $\mathrm{R}$ and $\mathrm{A}$ lines were transplanted @ 3-4 seedlings and 2 seedlings per hill, respectively. The row spacing maintained for R-R, R-A and A-A lines were 40, 30 and $15 \mathrm{~cm}$ respectively. Hill spacing for both $\mathrm{R}$ and $\mathrm{A}$ lines were maintained $15 \mathrm{~cm}$. Transplanting was done on different dates as per experimental treatments. Row directions were perpendicular to wind direction. Synchronization of flowering was adjusted at different panicle initiation stages by applying $2 \%$ phosphorus solution for earliness and 1\% nitrogen fertilizer solution for delay. Proper isolation was maintained through deployment of polythene barriers among the entries. Gibberellic acid $\left(\mathrm{GA}_{3}\right)$ at the rate of $220 \mathrm{~g} / \mathrm{ha}$ was used to improve panicle exertion and prolong duration of floret opening and stigma receptivity. Supplementary pollination was done with a bamboo stick at peak anthesis period for 3 to 4 times maintaining an interval 
of 30 minutes between them. Intercultural operation, irrigation and protection measures were maintained properly. Seed yield was harvested when it reached physiological maturity stage. Randomly ten hills were selected in each plot and the data were recorded on-days to first flowering, days to $50 \%$ flowering, days to maturity, plant height, panicle length, flag leaf length $(\mathrm{cm})$, number of tillers per plant, number of spikelets per panicle, number of filled spikelets per panicle, grain yield per plant, straw yield per plant, harvest index and percent out crossing rate. Data were analyzed using STAR computer software ANOVA-2 and Microsoft Excel programme 2007.

\section{RESULTS AND DISCUSSION}

The analysis of variance revealed significant differences among the tested hybrid combinations that indicated the seed production feasibility of hybrid rice combinations exist except for number of total spikelet's per panicle. It showed insignificant variation among the hybrid rice combinations under the present experiment (Table 1).

Table 2 shows the mean performance of hybrid combinations. The highest mean for plant height was found in IR75608A/BRRI20R $(97.07 \mathrm{~cm})$ which was followed by IR75608A/BRRI10R (96.37 cm) and IR75608A/BRRI31R (96.00 cm). The lowest mean for plant height was observed in BRRI11A/BRRI20R $(80.17 \mathrm{~cm})$ which was followed by BRRI10A/BRRI31R $(81.33 \mathrm{~cm})$.

Islam (2013) completely supported the findings of the present experiment when investigating approved hybrid rice genotypes for their seed quality assessment. The longest period for first flowering was found in IR79156A/BRRI15R (122.93 days) followed by IR75608A/BRRI15R (121.43 days) and IR79156A/BRRI10R (121.38 days),

Table 1. Analysis of variance for different characters in 20 combination rice hybrids $(\mathrm{A} \times \mathrm{R})$ during Boro $2017-18$.

\begin{tabular}{|c|c|c|c|c|c|c|c|c|c|c|c|c|c|c|}
\hline \multirow[b]{2}{*}{ 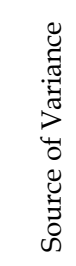 } & \multirow[t]{2}{*}{$\mathrm{DF}$} & \multicolumn{13}{|c|}{ Character } \\
\hline & & 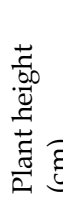 & 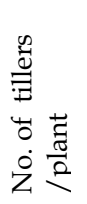 & 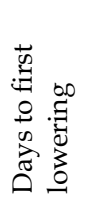 & 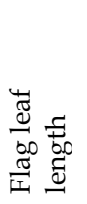 & 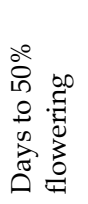 & 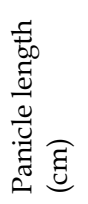 & 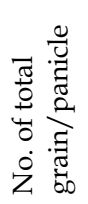 & 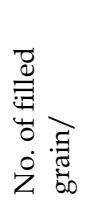 & 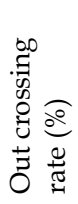 & 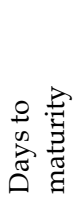 & 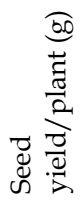 & 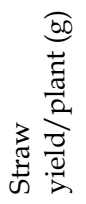 & 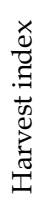 \\
\hline 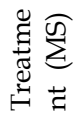 & $\stackrel{9}{7}$ & 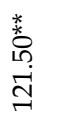 & 草 & 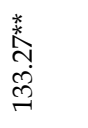 & 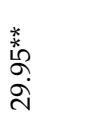 & 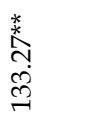 & $\underset{+}{\stackrel{*}{*}}$ & 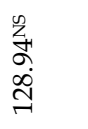 & $\begin{array}{l}* \\
* \\
0 \\
\ddot{O} \\
\ddot{n}\end{array}$ & 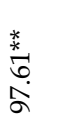 & 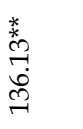 & 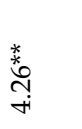 & 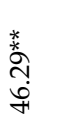 & $\begin{array}{l}\stackrel{*}{*} \\
\stackrel{+}{+} \\
\stackrel{10}{+}\end{array}$ \\
\hline 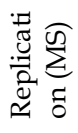 & $N$ & $\stackrel{ }{\check{C}}$ & $\begin{array}{l}\circ \\
\infty \\
\infty\end{array}$ & $\begin{array}{l}\text { مิ } \\
\text { ิે }\end{array}$ & $\stackrel{ }{ }$ & $\begin{array}{l}\text { ڤ̊ } \\
\text { ஸे }\end{array}$ & $\underset{+}{N}$ & 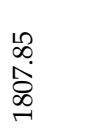 & तें & $\begin{array}{l}\hat{1} \\
\text { จุ }\end{array}$ & $\begin{array}{l}\text { ถุ } \\
\text { सं }\end{array}$ & $\underset{0}{\hat{~}}$ & 歺 & ஜ \\
\hline 宒 & $\infty$ & $\begin{array}{l}\hat{\sigma} \\
\text { ळ. } \\
0\end{array}$ & ڤ్ & $\underset{\sigma}{\sigma}$ & $\begin{array}{l}\widehat{\widehat{N}} \\
\text { ๙ }\end{array}$ & б్ర & 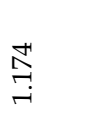 & $\begin{array}{l}\text { ิㅗ } \\
\text { ปุ } \\
\text { ปิ }\end{array}$ & $\begin{array}{l}\infty \\
\infty \\
\infty\end{array}$ & $\begin{array}{l}10 \\
\text { เి } \\
0\end{array}$ & $\stackrel{\widehat{N}}{\circ}$ & $\stackrel{\stackrel{1}{7}}{\overparen{0}}$ & 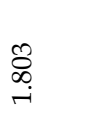 & $\begin{array}{l}\infty \\
\stackrel{1}{n} \\
\infty\end{array}$ \\
\hline$\vec{u}$ oீ & & $\underset{ت}{\stackrel{0}{0}}$ & $\begin{array}{l}\infty \\
\text { nె. }\end{array}$ & $\underset{\mathfrak{O}}{\mathbb{N}}$ & 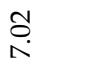 & gి & $\underset{+}{\stackrel{\infty}{+}}$ & $\begin{array}{l}0 \\
+1 \\
\infty\end{array}$ & ڤె & 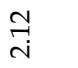 & 永 & గె & $\begin{array}{l}\infty \\
\stackrel{\infty}{\infty} \\
\end{array}$ & $\stackrel{\substack{N \\
N}}{ }$ \\
\hline
\end{tabular}

Legend: DF = Degree of freedom, NS = Insignificant and ** Significant at the $1 \%$ level of significance, CV= Co-efficient of variation. 
whereas BRRI7A/BRRI31R took the shortest time (102.93 days) which was followed by BRRI7A/BRRI20R (103.60 days). The longest period for $50 \%$ flowering was found in IR79156A/BRRI15R (128.27 days) followed by IR75608A/BRRI15R (126.77 days) and IR79156A/BRRI10R (126.71 days), on the other hand BRRI7A/BRRI10R and BRRI7A/BRRI31R took the shortest time (108.27 days) which was followed by BRRI7A/BRRI20R (108.93 days). Shikder (2010) found similar results while working with exotic hybrid rice genotypes in Boro season. Rice varieties in Boro season showed great fluctuations on flowering dates. These results are in close agreement with the previous findings by Roy (2006) and Ali 2007. The highest mean number of tillers per plant was recorded in BRRI7A/BRRI31R (17.33) which was followed by BRRI10A/BRRI20R (16.33) and BRRI7A/BRRI20R (16.17). The lowest mean number of tillers per plant was observed in IR75608A/BRRI10R (12.53) which was followed by IR75608A/BRRI20R (12.77) and IR75608A/BRRI15R (12.83). Rice plant generally produces more number of tillers than panicles. Unproductive tillers or the tillers that are produced at later dates and do not produce panicles before harvest are rather wasteful process (Mamin, 2003). The above findings were also supported by Saha (1998). Yadav (2001) also found similar trend in case of significant variability for number of tillers per plant in rice. The longest flag leaf length was found in IR75608A/BRRI15R $(31.37 \mathrm{~cm})$ which were followed by BRRI10A/BRRI20R $(29.80 \mathrm{~cm})$ and IR79156A/BRRI10R $(25.63 \mathrm{~cm})$. The shortest flag leaf length was recorded in IR75608A/BRRI10R $(15.97 \mathrm{~cm})$. Maximum number of panicles per plant was produced by BRRI7A/BRRI31R (14.00) which was followed by BRRI10A/BRRI31R (13.07), and BRRI11A/BRRI31R (12.47). The minimum number of panicle per plant was produced by IR75608A/BRRI15R (8.17) which was followed by IR75608A/BRRI10R (8.37). Islam (2013) somehow agreed with the present results. They found moderate number of panicle per plant with high genetic advance in percent of mean during working with developed hybrid rice genotypes. Rahman (2010) completely supported the findings of the present experiment while working with BRRI hybrid dhan2 variety for hybrid seed production.

The longest day to maturity was recorded 154.60 days in IR79156A/BRRI15R which was followed by IR75608A/BRRI15R (153.10 days). BRRI7A/BRRI10R took the shortest time (134.33 days) for maturation which was followed by BRRI7A/BRRI31R (134.67 days) and BRRI7A/BRRI20R (135.00 days). This feature indicated less influence of environment on the expression of this character. Hossain (2004) reported similar results of the present study, while working with 30 rice genotypes. Nath (2005) also supported the above findings for days to maturity in T. Aman rice varieties. The longest panicle length was observed in IR79156A/BRRI10R $(24.60 \mathrm{~cm})$ which was followed by IR75608A/BRRI20R $(24.57 \mathrm{~cm})$ and BRRI7A/BRRI10R $(24.40 \mathrm{~cm})$ respectively. The shortest panicle length was observed in IR75608A/BRRI10R $(21.17 \mathrm{~cm})$ which was followed by IR79156A/BRRI31R $(21.33 \mathrm{~cm})$. Bhandarkar et al, (2002) evaluated 52 genotypes of rice and reported high heritability for panicle length which supported the present outcomes. The highest number of total spikelets per panicle was found in genotype IR79156A/BRRI31R (148.87) which was followed by BRRI7A/BRRI10R (140.90). The lowest number of total spikelets per panicle was recorded in genotype BRRI11A/BRRI31R (119.97) which was followed by BRRI11A/BRRI20R.

Shikder (2010) completely supported the findings of the present experiment while investigating enormous exotic hybrid rice genotypes for their seed quality assessment. The highest value for percent out crossing rate (\%OCR) was produced by the combination BRRI7A/BRRI31R (50.23\%) which was followed by BRRI10A/BRRI10R (46.40\%). The lowest value for percent out crossing rate 
(\%OCR) was found in the combination of BRRI7A/BRRI15R (30.30\%) which was followed by BRRI7A/BRRI10R (32.47\%). The highest value for straw yield per plant was recorded in BRRI7A/BRRI31R (25.50 g) which was followed by BRRI10A/BRRI10R (24.60 g) and BRRI10A/BRRI15R (23.97 g) respectively. The lowest value for straw yield per plant was found in IR79156A/BRRI31R (12.20 g) which was followed by BRRI7A/BRRI15R (13.70 g). The highest value for harvest index was found in BRRI11A/BRRI15R (28.53\%) which was followed by BRRI7A/BRRI15R (28.16) and BRRI7A/BRRI10R (26.06\%) respectively. The lowest value for harvest index was recorded in BRRI10A/BRRI15R (17.02\%) which was followed by BRRI11A/BRRI10R (23.73\%). Iftekharudduala et al. (2001) found low GCV and PCV with high heritability and high genetic advance in percent per mean in irrigated rice which is more or less similar to the present study.

Table 2. Mean SD and SE value of seed yield contributing character of selected approved combinations of hybrid rice.

\begin{tabular}{|c|c|c|c|c|c|c|c|c|c|c|c|c|}
\hline Combination & $\begin{array}{l}\text { Plant } \\
\text { height } \\
(\mathrm{cm})\end{array}$ & $\begin{array}{l}\text { No. of } \\
\text { tillers / } \\
\text { plant }\end{array}$ & $\begin{array}{l}\text { No. of } \\
\text { panicle/ } \\
\text { plant }\end{array}$ & $\begin{array}{l}\text { Panicle } \\
\text { length } \\
(\mathrm{cm})\end{array}$ & $\begin{array}{l}\text { No. of } \\
\text { total } \\
\text { grain/ } \\
\text { panicle }\end{array}$ & $\begin{array}{l}\text { Out } \\
\text { crossing } \\
\text { rate }(\%)\end{array}$ & $\begin{array}{l}\text { Flag leaf } \\
\text { length } \\
(\mathrm{cm})\end{array}$ & $\begin{array}{l}\text { Straw } \\
\text { yield/pl } \\
\text { ant }(\mathrm{g})\end{array}$ & $\begin{array}{l}\text { Harvest } \\
\text { index }\end{array}$ & $\begin{array}{l}\text { Days to } \\
\text { first } \\
\text { flowering }\end{array}$ & $\begin{array}{l}\text { Days to } \\
50 \% \\
\text { flowering }\end{array}$ & $\begin{array}{l}\text { Days to } \\
\text { maturity }\end{array}$ \\
\hline $\begin{array}{l}\text { IR75608A/BR } \\
\text { RI10R }\end{array}$ & $96.37 \mathrm{a}$ & $12.53 \mathrm{f}$ & $8.37 \mathrm{i}$ & $21.17 \mathrm{f}$ & 129.63 & $33.50 \mathrm{~h}$ & $15.97 \mathrm{~h}$ & 15.44c-e & $25.55 a-c$ & $120.21 b-d$ & $125.55 b-d$ & $151.88 b-d$ \\
\hline $\begin{array}{l}\text { IR75608A/BR } \\
\text { RI15R }\end{array}$ & $94.33 \mathrm{~cd}$ & $12.83 \mathrm{ef}$ & $8.17 \mathrm{i}$ & $22.67 \mathrm{~b}-\mathrm{f}$ & 124.47 & $36.07 \mathrm{~g}$ & $31.37 a$ & $15.73 c-e$ & $24.95 \mathrm{c}$ & $121.43 \mathrm{~b}$ & $126.77 \mathrm{~b}$ & $153.10 \mathrm{~b}$ \\
\hline $\begin{array}{l}\text { IR75608A/BR } \\
\text { RI20R }\end{array}$ & $97.07 \mathrm{a}$ & $12.77 \mathrm{ef}$ & 8.60hi & $24.57 \mathrm{a}$ & 137.76 & $31.83 \mathrm{i}-\mathrm{k}$ & $21.77 \mathrm{fg}$ & $14.30 \mathrm{c}-\mathrm{f}$ & $24.88 \mathrm{c}$ & $119.93 \mathrm{~cd}$ & $125.27 \mathrm{~cd}$ & $151.60 \mathrm{~cd}$ \\
\hline $\begin{array}{l}\text { IR75608A/BR } \\
\text { RI31R }\end{array}$ & $96.00 \mathrm{ab}$ & $14.00 \mathrm{c}-\mathrm{f}$ & $9.30 \mathrm{~g}-\mathrm{i}$ & 23.27ae & 129.83 & $42.40 \mathrm{~d}$ & $23.81 \mathrm{~b}-\mathrm{f}$ & $20.00 \mathrm{~b}$ & $24.63 \mathrm{c}$ & 118.93d-f & $124.27 \mathrm{~d}-\mathrm{f}$ & 150.60d-f \\
\hline $\begin{array}{l}\text { IR79156A/BR } \\
\text { RI10R }\end{array}$ & $93.33 \mathrm{~cd}$ & $14.07 \mathrm{~b}-\mathrm{f}$ & $9.90 \mathrm{~d}-\mathrm{i}$ & $24.60 \mathrm{a}$ & 127.07 & $31.17 \mathrm{kl}$ & $25.97 \mathrm{~b}$ & 13.97d-f & $26.41 a-c$ & $121.38 \mathrm{~b}$ & $126.71 \mathrm{~b}$ & $153.05 \mathrm{~b}$ \\
\hline $\begin{array}{l}\text { IR79156A/BR } \\
\text { RI15R }\end{array}$ & $94.13 \mathrm{~cd}$ & $12.93 \mathrm{~d}-\mathrm{f}$ & $9.67 \mathrm{f}-\mathrm{i}$ & $21.53 \mathrm{ef}$ & 134.63 & $32.83 \mathrm{~h}-\mathrm{j}$ & $25.07 \mathrm{~b}-\mathrm{d}$ & $16.25 \mathrm{c}$ & $26.03 a-c$ & $122.93 a$ & $128.27 \mathrm{a}$ & $154.60 \mathrm{a}$ \\
\hline $\begin{array}{l}\text { IR79156A/BR } \\
\text { RI20R }\end{array}$ & 92.93de & $14.87 \mathrm{~b}-\mathrm{e}$ & 10.70c-h & $22.49 \mathrm{c}-\mathrm{f}$ & 135.27 & $41.07 \mathrm{e}$ & $22.63 \mathrm{~d}-\mathrm{g}$ & $20.67 \mathrm{~b}$ & $24.94 \mathrm{c}$ & $120.27 \mathrm{~b}-\mathrm{d}$ & $125.60 \mathrm{~b}-\mathrm{d}$ & $151.93 \mathrm{~b}-\mathrm{d}$ \\
\hline $\begin{array}{l}\text { IR79156A/BR } \\
\text { RI31R }\end{array}$ & $93.87 \mathrm{~cd}$ & $13.93 \mathrm{c}-\mathrm{f}$ & 9.77e-i & $21.33 \mathrm{f}$ & 148.87 & $31.67 \mathrm{jk}$ & $23.23 \mathrm{~b}-\mathrm{g}$ & $12.20 \mathrm{f}$ & $24.38 \mathrm{c}$ & $120.93 \mathrm{bc}$ & $126.27 b c$ & $152.60 \mathrm{bc}$ \\
\hline $\begin{array}{l}\text { BRRI7A/BRR } \\
\text { I10R }\end{array}$ & 92.90de & $15.50 \mathrm{a}-\mathrm{c}$ & $11.33 \mathrm{~b}-\mathrm{g}$ & $24.40 \mathrm{ab}$ & 140.90 & $32.47 \mathrm{~h}-\mathrm{j}$ & $25.63 \mathrm{bc}$ & $15.43 c-e$ & $26.06 a-c$ & $102.93 \mathrm{k}$ & $108.27 \mathrm{k}$ & $134.33 \mathrm{k}$ \\
\hline $\begin{array}{l}\text { BRRI7A/BRR } \\
\text { I15R }\end{array}$ & $93.17 \mathrm{~cd}$ & $15.33 a-c$ & $12.33 a-c$ & 21.87d-f & 132.98 & 30.301 & $24.90 \mathrm{~b}-\mathrm{d}$ & 13.70ef & 28.16ab & $104.60 \mathrm{j}$ & $109.93 \mathrm{j}$ & $136.00 \mathrm{j}$ \\
\hline $\begin{array}{l}\text { BRRI7A/BRR } \\
\text { I20R }\end{array}$ & $94.57 \mathrm{bc}$ & 16.17a-c & $12.00 \mathrm{a}-\mathrm{e}$ & $23.73 a-c$ & 129.89 & $33.60 \mathrm{~h}$ & $23.13 \mathrm{c}-\mathrm{g}$ & $15.63 c-e$ & $25.37 \mathrm{bc}$ & $103.60 \mathrm{jk}$ & $108.93 \mathrm{jk}$ & $135.00 \mathrm{jk}$ \\
\hline $\begin{array}{l}\text { BRRI7A/BRR } \\
\text { I31R }\end{array}$ & $91.60 \mathrm{e}$ & $17.33 a$ & $14.00 \mathrm{a}$ & $23.43 a-d$ & 135.26 & $50.23 a$ & $24.60 \mathrm{~b}-\mathrm{e}$ & $25.50 \mathrm{a}$ & $24.29 \mathrm{c}$ & $102.93 \mathrm{k}$ & $108.27 \mathrm{k}$ & $134.67 \mathrm{jk}$ \\
\hline $\begin{array}{l}\text { BRRI10A/BR } \\
\text { RI10R }\end{array}$ & $81.43 \mathrm{gh}$ & $15.83 a-c$ & $11.67 \mathrm{~b}-\mathrm{f}$ & $22.60 \mathrm{c}-\mathrm{f}$ & 129.13 & $46.40 \mathrm{~b}$ & $24.20 \mathrm{~b}-\mathrm{f}$ & $24.60 \mathrm{a}$ & $24.19 \mathrm{c}$ & $115.55 \mathrm{i}$ & $120.88 \mathrm{i}$ & $147.21 \mathrm{i}$ \\
\hline $\begin{array}{l}\text { BRRI10A/BR } \\
\text { RI15R }\end{array}$ & $83.03 \mathrm{f}$ & $15.33 a-c$ & $12.00 \mathrm{a}-\mathrm{e}$ & $21.60 \mathrm{ef}$ & 132.00 & $37.87 f$ & $23.70 \mathrm{~b}-\mathrm{g}$ & $23.97 \mathrm{a}$ & $17.02 \mathrm{~d}$ & 117.07gh & 122.4gh & 148.73gh \\
\hline $\begin{array}{l}\text { BRRI10A/BR } \\
\text { RI20R }\end{array}$ & $82.67 \mathrm{fg}$ & $16.33 \mathrm{ab}$ & $12.17 a-d$ & $22.00 \mathrm{c}-\mathrm{f}$ & 138.96 & $35.53 \mathrm{~g}$ & $29.80 \mathrm{a}$ & $16.13 \mathrm{~cd}$ & $23.95 c$ & 116.93gh & $122.27 \mathrm{gh}$ & 148.60gh \\
\hline $\begin{array}{l}\text { BRRI10A/BR } \\
\text { RI31R }\end{array}$ & $81.33 \mathrm{gh}$ & $15.90 \mathrm{a}-\mathrm{c}$ & 13.07ab & $22.13 c-f$ & 133.07 & $32.53 \mathrm{~h}-\mathrm{j}$ & $21.77 \mathrm{fg}$ & $14.50 \mathrm{c}-\mathrm{e}$ & $24.59 \mathrm{c}$ & 116.40hi & 121.73hi & 148.07hi \\
\hline $\begin{array}{l}\text { BRRI11A/BR } \\
\text { RI10R }\end{array}$ & 82.00fg & $15.20 \mathrm{a}-\mathrm{d}$ & $11.03 \mathrm{~b}-\mathrm{g}$ & 21.93d-f & 134.50 & 32.97hi & $22.90 \mathrm{c}-\mathrm{g}$ & $14.20 \mathrm{c}-\mathrm{f}$ & $23.73 c$ & $117.93 \mathrm{e}-\mathrm{g}$ & $123.27 \mathrm{e}-\mathrm{g}$ & $149.60 \mathrm{e}-\mathrm{g}$ \\
\hline $\begin{array}{l}\text { BRRI11A/BR } \\
\text { RI15R }\end{array}$ & $82.17 \mathrm{fg}$ & $14.17 \mathrm{~b}-\mathrm{f}$ & $10.67 \mathrm{c}-\mathrm{h}$ & $22.30 c-f$ & 128.80 & $44.80 \mathrm{c}$ & $20.97 \mathrm{~g}$ & $19.97 \mathrm{~b}$ & $28.53 a$ & 118.93d-f & $124.27 \mathrm{~d}-\mathrm{f}$ & $150.60 d-f$ \\
\hline $\begin{array}{l}\text { BRRI11A/BR } \\
\text { RI20R }\end{array}$ & $80.17 \mathrm{~h}$ & $15.27 a-c$ & $11.43 \mathrm{~b}-\mathrm{g}$ & 21.90d-f & 123.60 & $32.33 \mathrm{~h}-\mathrm{k}$ & $22.77 d-g$ & 15.43c-e & $24.07 \mathrm{c}$ & 119.27de & $124.60 \mathrm{de}$ & 150.93de \\
\hline $\begin{array}{l}\text { BRRI11A/BR } \\
\text { RI31R }\end{array}$ & $81.67 f-h$ & $15.77 a-c$ & $12.47 a-c$ & $22.03 \mathrm{c}-\mathrm{f}$ & 119.97 & $36.67 \mathrm{fg}$ & $22.03 \mathrm{e}-\mathrm{g}$ & $14.37 \mathrm{c}-\mathrm{f}$ & $24.34 \mathrm{c}$ & $117.60 \mathrm{f}-\mathrm{h}$ & $122.93 \mathrm{f}-\mathrm{h}$ & $149.27 \mathrm{f}-\mathrm{h}$ \\
\hline Mean & 89.24 & 14.80 & 10.93 & 22.58 & 132.28 & 36.31 & 23.81 & 17.10 & 24.80 & 115.99 & 121.32 & 147.62 \\
\hline SD & 6.33 & 1.81 & 2.00 & 1.42 & 13.55 & 5.87 & 3.16 & 4.04 & 2.85 & 6.66 & 6.65 & 6.69 \\
\hline SE & 1.415 & 0.405 & 0.447 & 0.318 & 3.030 & 1.313 & 0.707 & 0.903 & 0.637 & 1.489 & 1.487 & 1.496 \\
\hline $\mathrm{CV} \%)$ & 1.04 & 9.38 & 12.55 & 4.80 & 8.46 & 2.12 & 13.27 & 7.85 & 7.28 & 0.717 & 0.685 & 0.554 \\
\hline
\end{tabular}

Legend: $\mathrm{SD}=$ Standard deviation (SD), $\mathrm{SE}=$ Standard error and $\mathrm{CV}=\mathrm{Co}$-efficient of variation. 
The highest number of filled spikelets per panicle was produced by BRRI7A/BRRI31R (48.20) which was followed by IR79156A/BRRI31R (47.83) and BRRI7A/ BRRI10R (44.63) respectively. Table 3 shows the lowest number of filled spikelets per panicle exhibited by BRRI11A/BRRI31R (36.27) which was followed by BRRI10A/ BRRI15R (36.37). Almost similar result was quoted by Bidhan et al. (2001) who reported high heritability coupled with high genetic advance for number of filled spikelet's per panicle.

The highest value for grain yield per plant was recorded in BRRI7A/BRRI31R (8.17g) which was followed by BRRI10A/ BRRI10R (7.85 g) and BRRI11A/BRRI15R
(7.68 respectively. Table 3 shows the lowest value for grain yield per plant found in IR79156A/BRRI31R (3.97 g) which was followed by BRRI11A/ BRRI10R (4.44 g). Similar report was presented by Shrirame and Muley (2003) who worked out high co-efficient of variability with high heritability for grain yield per plant in hybrid rice. Mishra and Verma (2002) also reported high heritability coupled with high genetic advanced for yield per plant in rice. Iftekharudduala et al. (2001) reported high variation among genotypes in respect of the trait which is similar to the present study. Roy (2006) also expressed similar agreement with the results of the present study.

Table 3. F1 seed production potentiality of different CMS lines crossed with restorer lines.

\begin{tabular}{lcc}
\hline Combination & No. of filled grain/panicle & F $_{1}$ seed yield per plant $(\mathrm{g})$ \\
\hline IR75608A/BRRI10R & $40.40 \mathrm{~b}-\mathrm{d}$ & $5.29 \mathrm{c}-\mathrm{f}$ \\
IR75608A/BRRI15R & $37.73 \mathrm{~cd}$ & $5.23 \mathrm{c}-\mathrm{f}$ \\
IR75608A/BRRI20R & $39.13 \mathrm{~cd}$ & $4.74 \mathrm{e}-\mathrm{h}$ \\
IR75608A/BRRI31R & $38.53 \mathrm{~cd}$ & $6.54 \mathrm{~b}$ \\
IR79156A/BRRI10R & $38.67 \mathrm{~cd}$ & $5.01 \mathrm{~d}-\mathrm{h}$ \\
IR79156A/BRRI15R & $40.87 \mathrm{~b}-\mathrm{d}$ & $5.71 \mathrm{c}$ \\
IR79156A/BRRI20R & $40.40 \mathrm{~b}-\mathrm{d}$ & $6.87 \mathrm{~b}$ \\
IR79156A/BRRI31R & $47.83 \mathrm{a}$ & $3.97 \mathrm{i}$ \\
BRRI7A/BRRI10R & $44.63 \mathrm{ab}$ & $5.42 \mathrm{~cd}$ \\
BRRI7A/BRRI15R & $36.63 \mathrm{~cd}$ & $5.37 \mathrm{~cd}$ \\
BRRI7A/BRRI20R & $37.33 \mathrm{~cd}$ & $5.31 \mathrm{c}-\mathrm{e}$ \\
BRRI7A/BRRI31R & $48.20 \mathrm{a}$ & $8.17 \mathrm{a}$ \\
BRRI10A/BRRI10R & $38.60 \mathrm{~cd}$ & $7.85 \mathrm{a}$ \\
BRRI10A/BRRI15R & $36.37 \mathrm{~cd}$ & $4.90 \mathrm{~d}-\mathrm{h}$ \\
BRRI10A/BRRI20R & $41.20 \mathrm{bc}$ & $5.07 \mathrm{~d}-\mathrm{g}$ \\
BRRI10A/BRRI31R & $40.63 \mathrm{~b}-\mathrm{d}$ & $4.72 \mathrm{f}-\mathrm{h}$ \\
BRRI11A/BRRI10R & $39.30 \mathrm{~cd}$ & $4.44 \mathrm{hi}$ \\
BRRI11A/BRRI15R & $38.53 \mathrm{~cd}$ & $7.68 \mathrm{a}$ \\
BRRI11A/BRRI20R & $39.37 \mathrm{~cd}$ & $4.90 \mathrm{~d}-\mathrm{h}$ \\
BRRI11A/BRRI31R & $36.27 \mathrm{~d}$ & $4.63 \mathrm{gh}$ \\
\hline Mean & 40.03 & 5.59 \\
SD & 4.14 & 1.21 \\
SE & 0.926 & 0.271 \\
CV & 7.36 & 6.32 \\
\hline
\end{tabular}

Legend: $\mathrm{SD}=$ Standard deviation $(\mathrm{SD}), \mathrm{SE}=$ Standard error and $\mathrm{CV}=\mathrm{Co}$-efficient of variation. 
Seed production feasibility of hybrid rice combination in Boro season 2017-18, BRRI7A/BRRI31R hybrid rice combination showed considerable positive significant variation over an average in respect of yield and desirable variation for earliness in maturity.

\section{REFERENCES}

AIS. 2018. Krishi Diary 2016, Agricultural Information Service, Khamarbari, Farmgate, Dhaka 1215, Bangladesh.

Ali, M. 2007. Development of parental lines for aromatic hybrid rice. PhD Dissertation, Bangabandhu Sheikh Muzibur Rahman Agricultural University, Gazipur1706, Bangladesh. 219 p.

BBS, 2018. 45 years Agriculture Statistics of Major Crops. Statistics and Informatics

Bhandarkar, S V, Ravindra, K Arvind, R Verma and A Kumar. 2002. Genetic variability and correlation analysis in short duration rice. Pl. Archives. 2(1): 9598.

Bidhan, R, M Hossain, F Hossain and B Roy. 2001. Genetic variability in yield components of rice (Oryza sativa L.). Env. And Ecol. 19(1): 186-189.

Hossain, M B. 2004. Study of variability and genetic diversity in rice. MS thesis Department of Genetics and Plant Breeding. Bangladesh Agricultural University, Mymensingh.100 p.

Iftekharuddaula, K M, M A Badshah, M S Hassain, M K Bashar and K Akhter. 2001. Genetic viability, character association and path analysis of yield components in irrigated rice (Oryza sativa L.). Bangladesh J. PI. Breed Genet. 14 (1): 43-49.
Islam, M R. 2013. Genetic Purity of Rice Hybrids Using Morphological and Molecular Markers. Ph.D. Thesis. Deptt. of Seed Science and Technology. BSMRAU, Salna, Gazipur.

Mamin, M S I. 2003. Photosynthesis, shoot reserve translocation, lodging and nitrogen use efficiency of modern-and traditional varieties of rice. Dissertation research. Bangabandhu Sheikh Mujibur Rahman Agricultural University, Salna, Gazipur 1706. 272 p.

Mishra, L K and P K Verma. 2002. Genetic variability for quality and yield traits in nonsegregating populations of rice (Oryza sativa L.). Pl. Archives. 2(2): 251-256.

Nath, J K. 2005. Study of genetic divergence in T.Aman rice. MS Thesis, Department of Genetics and Plant Breeding. Bangabandhu Sheikh Mujibur Rahman Agricultural University, Gazipur-1706, Bangladesh. $70 \mathrm{P}$.

Rahman, M H, M H Ali, M J Hasan, M U Kulsum and M M Khatun. 2010. Outcrossing rate in row ratio of restorer and CMS lines for hybrid rice seed production. Eco-friendly Agril. J. 3(5): 233-236.

Roy, A K. 2006. Development of aromatic cytosource for hybrid rice production. PhD Dissertation, Bangabandhu Sheikh Muzibur Rahman Agricultural University, Gazipur 1706, Bangladesh. 166 p.

Saha, S. 1998. Yield and quality characters of local and modern rice cultivars. MS thesis, Department of Genetics and Plant Breeding, IPSA, Gazipur-1706. 76 p.

Shikder, M N I. 2010. Seed quality assessment in hybrid rice genotypes. PhD Dissertation, Bangabandhu Sheikh Muzibur Rahman Agricultural University, Gazipur-1706, Bangladesh. 187 p.

Shrirume, M D and D M Muley. 2003. Variability and correlation studies in rice (Oryza sativa L.). J. Soils and Crops. 13 (1):165-167.

Yadav, R K. 2001.Variation in local accessions of rice collection under rainfed condition in Chattisgarh. Indian J. Dry land Agril.Res. Dev. 16(2): 168-169. 
